\title{
Comparative Analysis of the Thermal Resistance Profiles of Power Light-Emitting Diodes Cree and Rebel Types
}

\author{
A.L. Zakgeim ${ }^{1}$, A.E. Chernyakov ${ }^{1}$, A.S. Vaskou ${ }^{2}$, V.K. Kononenko ${ }^{2}$, V.S. Niss ${ }^{2}$ \\ ${ }^{1}$ Submicron Heterostructures for Microelectronics Research \& Engineering Center, RAS \\ 26 Polytekhnicheskaya Str., 194021 St. Petersburg, Russia \\ ${ }^{2}$ Belarusian National Technical University \\ 65 Independence Ave., 220013 Minsk, Belarus \\ e-mails: vklavik@gmail.com; zakgeim@mail.ioffe.ru
}

\begin{abstract}
Analysis of the thermal resistance of power lightemitting diodes (LEDs) of Cree and Rebel types is developed. Components of the thermal resistance of the diodes are determined and several distinguishes between different methods are obtained. Behavior of bottleneck effect related to definite interfaces is established. The value of LED active junction area is evaluated too.
\end{abstract}

\section{Introduction}

For analysis and identification of the thermal resistance distribution and the value of $R_{\mathrm{jc}}$ in LEDs it is necessary to know their detail construction with the sizes of elements. Comparison of measured thermal parameters has been given earlier for new types LEDs, which were produced by different technologies, i.e., by thin-film flip-chip (TFFC) - Rebel and ThinGaN of (vertical thin-flip) VTF structure with $\mathrm{Si}$ substrate Cree [1]. In the Rebel structure, in contrast to the Cree structure, a semiconductor or the sapphire substrate is not used.

The die for the Rebel LEDs in the TFFC technology is the GaN chip with a thickness of 5-6 $\mu \mathrm{m}$ planted on ceramic metal core printed circuit board (MCPCB). Calculated thermal resistance of such a $\mathrm{GaN}$ layer is the order of $0.05 \mathrm{~K} / \mathrm{W}$, thermal time constant is about of $0.5 \mu \mathrm{s}$, which beyond the resolving limit of T3Ster equipment applied in [1]. Thus, far we do not know exact sizes and materials of a die structure its thermal parameters identification will be difficult.

Detail thermal study of flip-chip LEDs characteristics can be produced, together with T3Ster measurements, using method of IR-thermovision microscopy [2]. Also, IR-thermovision microscopy allows to detect microdefects causing current leakage channels and potentially dangerous in terms of reliability of the devices.

Additional method of LED characterization consists in investigations of transient electrical processes in the diode sources under heating by direct current. The time dependence of the temperature of the active region of a source provides discrete and differential spectra of thermal impedance resistance [3]. By the developed method, thermal resistances of internal elements of the heterolasers and LEDs are determined.

There are questions also to the interface of direct bonded copper (DBC) and MCPCB layers. Here, for the precise estimation of the complete internal resistance $R \mathrm{jc}$ it can be used a recent method "Transient Dual Interface Measurement" introduced into the standard JESD51-14 [4]. The standard is applied first of all for thermal characterization of transistor devices.

\section{Measurement Results}

A thermal resistance characterization of different LEDs types has been developed by a T3Ster equipment and comparison analysis has been established with thermal relaxation spectrometry data. Obtained by T3Ster differential structure functions for Cree XPE and Luxeon Rebel ES LEDs types are presented in Fig. 1.

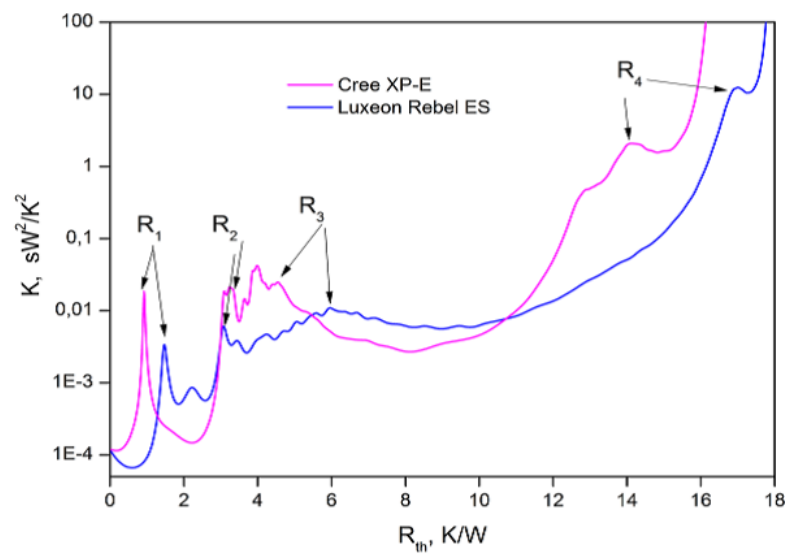

Figure 1: Differential structure functions of Cree XPE and Luxeon Rebel ES LEDs measured by T3Ster.

Measurements of thermal characteristics for different type LEDs obtained by T3Ster equipment were produced at various operation currents 200 to $1200 \mathrm{~mA}$. At increasing the operation current essential variations are related with thermal resistance of layer components near substrate where important heat spreading and effusion occur. This conclusion is supported by analysis of cumulative structure functions according to T3Ster methodic.

The principal differences of powerful Rebel and Cree LEDs consist in design of chip emitter, materials of substrate and DBC and MCPCB layers, configuration of bonding contacts, and sizes of active area as well. Images in own radiation of diode chips (Fig. 2) illustrate characteristic peculiarities of the LED configuration. 


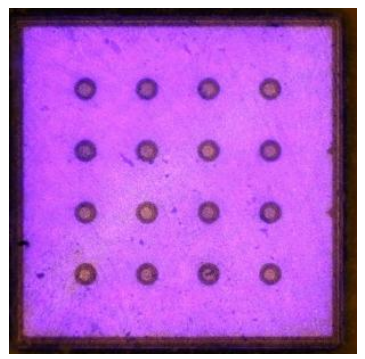

(a)

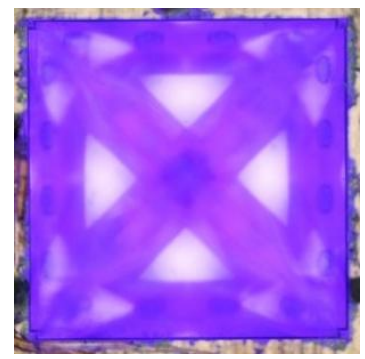

(b)
Figure 2: Images (a) of Rebel and (b) Cree XPE-HEW diode chips of $1 \mathrm{~mm}^{2}$ area without lens and luminescent powder in own rays. Metalized n-contacts are circle points and visible around the perimeter of the crystals.

Five types of LEDs were analyzed (Fig. 3). Both Rebel and Rebel ES diodes have flip chip mesa structure that bonded on ceramic MCPCB substrate, base of $\mathrm{DBC}$ is $\mathrm{Al}_{2} \mathrm{O}_{3}$, area of LED chip equils $S_{\text {ch }}=1 \mathrm{~mm}^{2}$ (Rebel) or $S_{\mathrm{ch}}=2 \mathrm{~mm}^{2}$ (Rebel ES). Cree samples with VTF structure are designed on $\mathrm{Si}$ substrate and two materials $\mathrm{DBC}$ are used, i.e., $\mathrm{Al}_{2} \mathrm{O}_{3}$ DBC for Cree XPE and AlN DBC for Cree XPG. The quantity of $S_{\mathrm{ch}}$ was $1 \mathrm{~mm}^{2}$ for the first unit and $2 \mathrm{~mm}^{2}$ for the second one. Another powerful LEDs are Cree XPE-HEW type with direct attachment on AIN DBC. These diodes have on top SiC substrate and area chip is $S_{\text {ch }}=1 \mathrm{~mm}^{2}$.

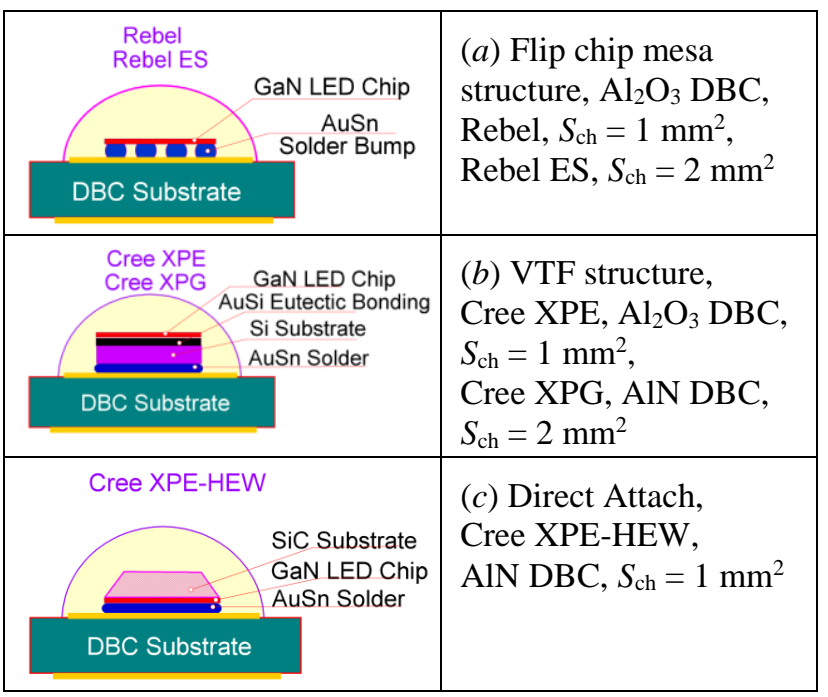

Figure 3: Schematic structures (a) of Rebel and Rebel ES, (b) Cree XPE and XPG, and (c) Cree XPE-HEW LEDs with different DBC materials and sizes.

General cumulative structure functions of different Rebel and Cree LEDs are presented in Fig. 4. The curves have been analyzed according to T3Ster methodic. Similar behavior for approached design and constructions of the LEDs are clearly visible. Here, data for Cree XPG and Rebel ES LEDs are normalized to area $1 \mathrm{~mm}^{2}$, i. e., values of thermal resistance $R_{\mathrm{th}}$ multiplied by two and of thermal capacitance $C_{\text {th }}$ divided by two. The profile curves are grouped at three diodes technologies (design) and two types of ceramic (AlN and $\mathrm{Al}_{2} \mathrm{O}_{3}$ ) substrates DBC.

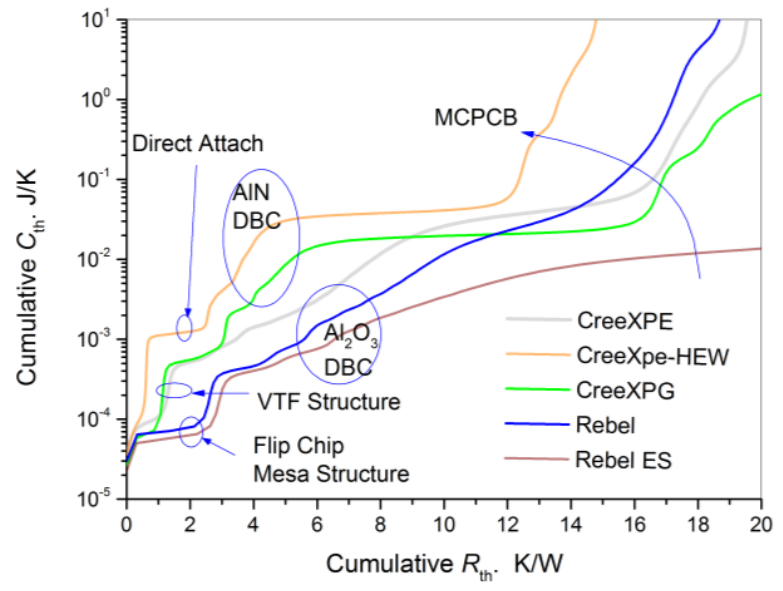

Figure 4: General cumulative structure functions of different Rebel and Cree LEDs.

Obviously, for correct thermal identification of complex diode structure must be to take into account in addition to $R_{\text {th }}$ the profile of $C_{\text {th. }}$. To analyze phenomena of definite pikes at the $K$ structure, it is useful to calculate at first a model cumulative structure function and then to determine its form by achievable methods (Fig. 5).

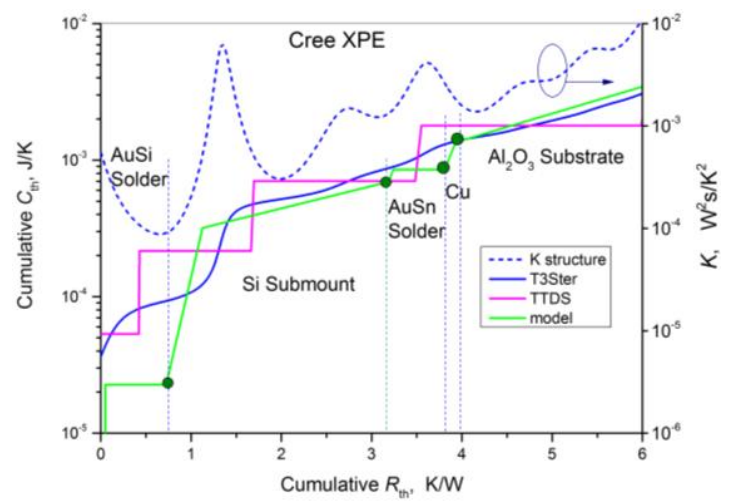

(a)

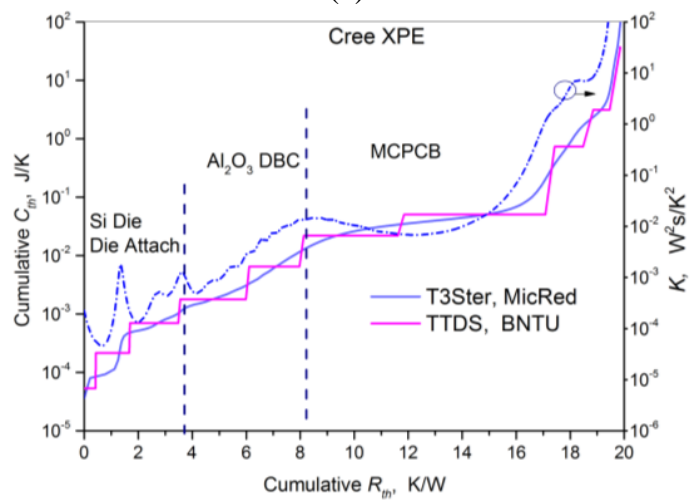

(b)

Figure 5: (a) Cumulative structure functions and $K$ function of a Cree XPE LED indicating various components of the die structure. (b) The functions extended up to including MCPCB are obtained by a T3Ster equipment and BNTU TTDS methodic. 
We have analyzed the structure functions by the T3Ster method and by Transient Thermal Differential Spectrometry (TTDS) developed in $[3,5,6]$. An example for Cree XPE is presented in Fig. 5 and also for Cree XPE-HEW in Fig. 6.

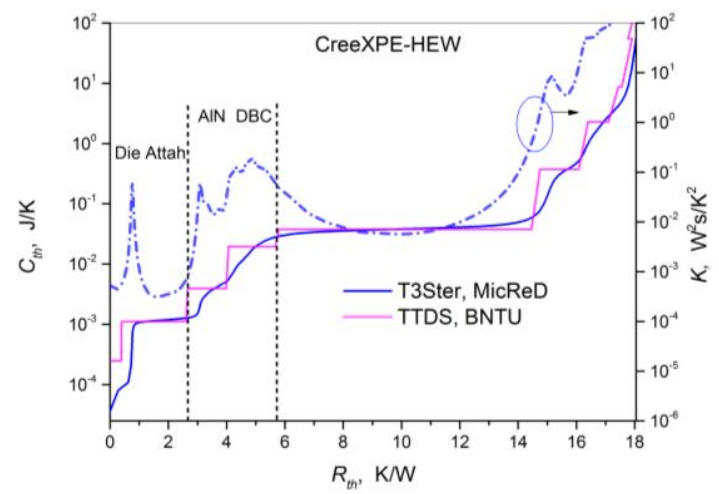

Figure 6: Cumulative structure functions and $K$ function of a Cree XPE-HEW LED indicating various components of the die structure. The functions extended up to MCPCB layer are obtained by a T3Ster equipment and BNTU TTDS methodic.

\section{Analysis in the Framework of the Electro- thermal Model}

Heating temperature $\Delta T$ of the device active region is determined by the total thermal resistance of its elements and inter-elements connections between $p-n$ junction and heat sink. According to electro-thermal analogy, transient processes in the semiconductor structure are described in the framework of the equivalent circuits [3] in the form of $R C$ chains as Foster and/or Cauer schemes. When cooling unit the overheat temperature dependence $\Delta T(t)$ should be a simple law, i. e.,

$$
\Delta T(t)=P_{T} \sum_{i}^{n} R_{i} \exp \left(-t / \tau_{i}\right) .
$$

Here, $P_{T}$ is the thermal power dissipation in device, $R_{i}$ is the thermal resistance of structural element $i$, $\tau_{i}=R_{i} C_{i}$ is the thermal relaxation time constant, $C_{i}$ is the heat capacitance of the element diode construction and heat sink design element, $n$ is the number of components.

In general, the thermal resistance of the structure is $R_{T}=\sum_{i}^{n} R_{i}$, where $n$ specifies the total number of structural components of the device. Dynamic thermal resistance is defined as $R^{*}(t)=\Delta T(t) / P_{T}$ and obtained from a functional dependency [3] in the form

$$
R^{*}(t)=\sum_{i}^{n} R_{i} \frac{t}{\tau_{i}} \exp \left(1-t / \tau_{i}\right) .
$$

As seen, in the case where the difference between thermal relaxation times constant $\tau_{i}$ for the nearest components of the structure is large enough the value of $R^{*}(t)$ at $t \approx \tau_{i}$ gives the value of thermal resistance $R_{i}$ for component $i$.
However, for complex multiple-layer structures, which modern semiconductor devices contain, function (2) is little informative. So, instead of differential dependence (2) it is acceptable to apply a higher-order function, for example, order $m$, in the form [6]

$$
R_{m}^{*}(t)=\sum_{i}^{n} R_{i}\left(t / m \tau_{i}\right)^{m} \exp \left(m\left(1-t / m \tau_{i}\right)\right) .
$$

The transition from (2) to (3) allows using numerical measurements pick up more precisely the thermal parameters of structural elements. This technique gives possibility to specify parameters $R_{i}$ and $\tau_{i}$ of the components.

A sufficient order $m=3$ and consequently dependence (3) for submission to the differential spectra of the thermal resistance is $R_{3}^{*}(t)$. When specify a sufficient large numbers of $n$ structural components, it can define a discrete spectrum of thermal resistance.

Typically, the number of thermal structural elements is determined by the software simulators or by efficiency of non-stationary thermal testers and limited to $n=3 \div 5$ (due to technical difficulties at measurements and complexity analysis) [1, 4, 7]. Advanced differential technique makes it possible to expand the number of thermal structural elements to $n=10$.

Detail analysis of thermal resistance profile in the LEDs is provided by a methodic of the thermal relaxation spectrometry $[3,5,6]$. As an example, data for Rebel and Rebel ES LEDs are presented in Fig. 7. There are 10-component $R C$ circuits in Cauer network. As seen, the highest values 3.44 and $5.40 \mathrm{~K} / \mathrm{W}$ of the thermal resistance belong to layers at the interface DBC-MCPCB. Therewith, time constant relaxation is of 40-70 ms. Consideration of the active area where heat flow distributes shows that behavior such a bottleneck appears at this region, principally for Rebel ES samples.

As concerns relaxation thermal spectrometry was approved using a device "Relaxation Impedance Spectrometer of Thermal Processes" [5]. The thermal characterization includes analysis of thermal $R C$ equivalent circuits (Foster and/or Cauer models), where $R_{\text {th }}$ is the thermal resistance and $C_{\text {th }}$ is the heat capacitance of the source layer elements.

By the developed method, the time dependence of the heating temperature of the device active region $\Delta T(t)$ (time resolution is of $2 \mu \mathrm{s}$ ) is obtained from the direct bias measured by an A/D converter (16-bit resolution) under a unit-step current pulse. Thermal resistances of internal elements of the LEDs are determined from the measured $\Delta T(t)$ dependence, values of the layer thermal resistance $R_{\mathrm{th}}$ and thermal time constant $\tau=R_{\mathrm{th}} C_{\mathrm{th}}$ are obtained. The same conclusions are fulfilled from analysis of the data measurements of Cree XPE and Cree XPG units with VTF structure (Fig. 8). 


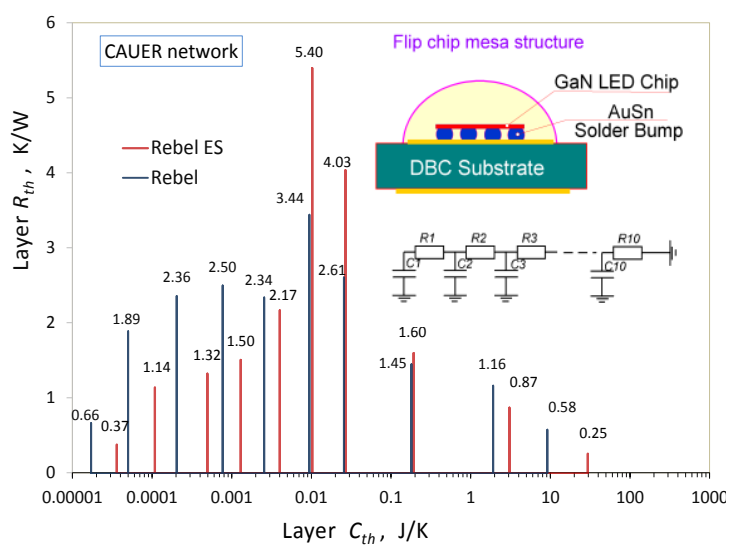

(a)

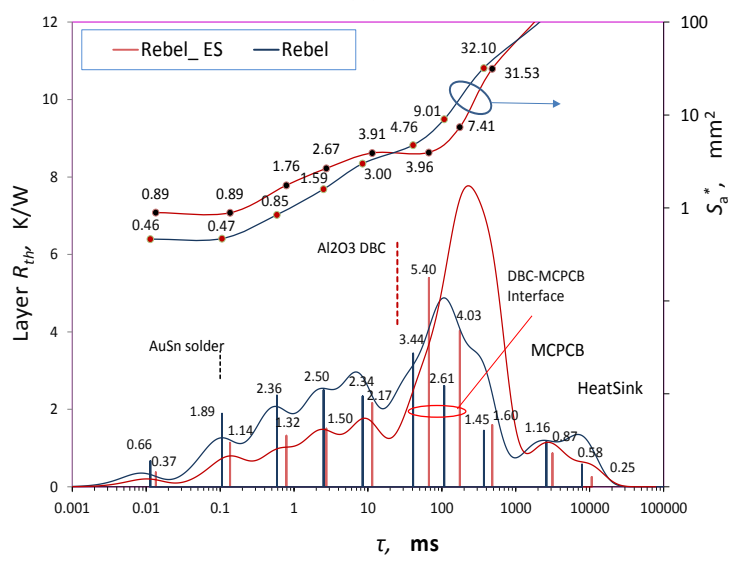

(b)

Figure 7: (a) Discrete thermal resistance spectra of Rebel and Rebel ES LED samples in the scale $R_{t h}-C_{t h}$. (b) Discrete and differential spectra $R_{t h}(\tau)$.

The bottleneck effect the most manifested for Cree XPG design where the layer $R_{\mathrm{th}}$ is of $6.57 \mathrm{~K} / \mathrm{W}$. So, in spite of developed effective ceramic based packages, the thermal dissipation bottleneck effect should be paid special attention and it requires additional investigations and new searches of more appropriate materials and design.

From Fig. 5, it is seen that the estimated and measured profiles match well to $C_{\text {th }}$. Falls only AuSi point for Cree XPE diode. This may be due to the limitations of the device T3Ster since characteristic thermal time of the AuSi layer with thickness of $10 \mu \mathrm{m}$ is less than $10 \mu \mathrm{s}$. The measured resistances $R_{\mathrm{th}}$ of solder layers AuSn and AuSi are in one and half or twice above calculated ones that apparently is connected with defects in the solders or with thermal barriers at the interface ends.

Special shape of the thermal profile is for Cree XPE-HEW structures. In these diodes, substrate $\mathrm{SiC}$ is outside and its thermal capacitance is more than 10 times higher than for AuSn layer, which increases the thermal inertia of the diode, and thermal resistance of the layer $\mathrm{SiC}$ does not contribute to the stationary thermal profile. Notable good correlation $R_{\text {th }}$ of AuSn layer for diodes Rebel and Cree HPE-HEW. Mention, that we take into account the effective area of chip $S_{\mathrm{a}}{ }^{*}$ including the fill factor $\gamma$, so $S_{\mathrm{a}}{ }^{*}=\gamma S_{\mathrm{ch}}$. For Rebel structures where gold bumps are used $\gamma \approx 0.45$ and for Cree structures $\gamma \approx 0.65$. In addition, through thermal profiles $R_{\mathrm{th}} / C_{\mathrm{th}}$ we can also build profiles of temperature gradients in statics and dynamics and assess their impact on reliability of LEDs.

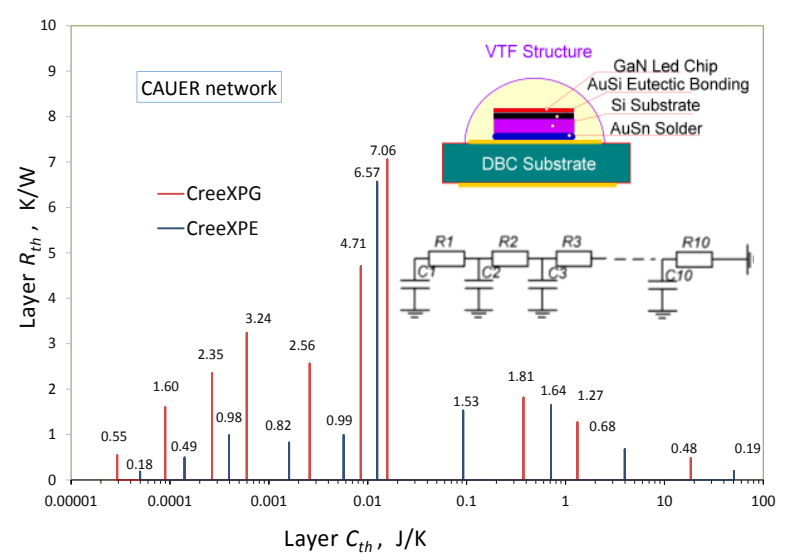

(a)

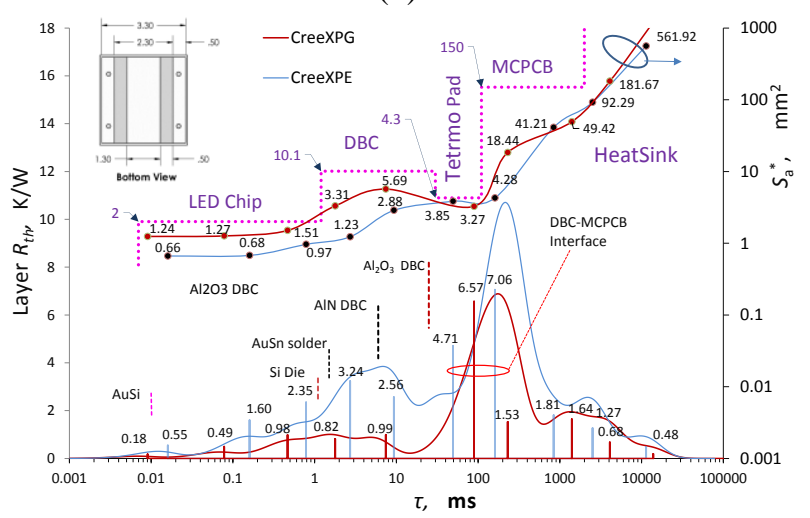

(b)

Figure 8: (a) Discrete thermal resistance spectra of Cree XPE and Cree XPG samples in the scale $R_{t h}-C_{t h}$. (b) Discrete and differential spectra $R_{t h}(\tau)$.

\section{Initial Plot Heating and the Device Active Area}

As identified [7], for a planar structure, where heat propagates perpendicular to the surface of the device, the active region of which is heated with constant power density $P / S$, the region temperature $\Delta T$ rises linearly with the square root of heating time $t$, i.e., $\Delta T(t)=2 P t^{1 / 2} / S_{\mathrm{a}}\left(\pi \kappa \rho c_{p}\right)^{1 / 2}$. Here, $c_{p}, \rho$, and $\kappa$ are the specific heat, density, and thermal conductivity of the chip material, respectively.

This conclusion follows from the solution of unsteady heat conduction equation that has the form [8]

$$
\begin{aligned}
& T(t)=P R_{T}\left(2 \sqrt{t / \pi \tau}+\sum_{n=1}^{\infty} 4(-1)^{n} \times\right. \\
& \left.\times\left(\sqrt{t / \pi \tau} \mathrm{e}^{-n^{2} \tau / t}-n(1-\operatorname{erf}(n / \sqrt{t / \tau}))\right)\right)
\end{aligned} .
$$

During the initial plot, heating of the device active region is directly proportional to $t^{1 / 2}$, i.e., 


$$
\Delta T(t)=\frac{2 P R_{T}}{\sqrt{\pi}} \frac{\sqrt{t}}{\sqrt{\tau}},
$$

where the thermal relaxation time-constant $\tau=\left(R_{\mathrm{th}} S_{\mathrm{a}}\right)^{2} \kappa c_{p} \rho$ and $R_{\mathrm{th}}$ is the thermal resistance of the active layer of the area $S_{\mathrm{a}}$.

For example, Si substrate with thickness of $300 \mu \mathrm{m}$ provides the thermal relaxation constant $\tau$ of $1-3 \mathrm{~ms}$ and the initial heating plot corresponds to the time $t \leq 0.4 \tau$ (Fig. 9). In exponential approach $T(t)$ initially follows proportionally $t[3,9]$.

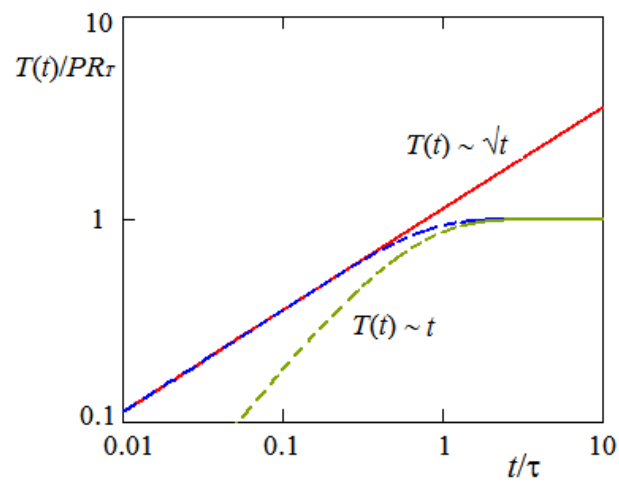

Figure 9: Change in the temperature $T(t)$ of the device active area. The initial heating plot is directly proportional to $t^{1 / 2}$ and coincides with the exact solution (1) unlike linear relationship $T(t) \sim t$.

From the measured function $\Delta T(t)$ can easily be found the quantity of time degree $n^{*}$ of a heating law. An additional benefit is a possibility to estimate the chip area $S_{\mathrm{a}}[4,10,11]$. Results of determining the active area $S_{\mathrm{a}}$ and time degree $n^{*}$ of the temperature rise law indicate that the square root time dependence under heating process is provided for the LEDs in the interval $0.01-0.1 \mathrm{~ms}$ (Rebel ES) and 0.1 to $1 \mathrm{~ms}$ (Cree $\mathrm{XPG})$. The active area for these LEDs types is about of $2 \mathrm{~mm}^{2}$.

Another presentation and determination of the active area is a method using the term $e$ as thermal effusivity, or the term $\alpha=\kappa / \rho c_{p}$ as thermal diffusivity at die layer components. If obtain a relation between thermal characteristics $R_{\text {th }}$ and $C_{\text {th }}$ of layer components of LEDs through the quantity

$$
R_{\mathrm{th}} / C_{\mathrm{th}}=\frac{\alpha}{\kappa^{2} S_{\mathrm{a}}^{2}}=\frac{1}{\kappa \rho c_{p} S_{\mathrm{a}}^{2}},
$$

we can to examine variation of the active area $S_{\mathrm{a}}$ duration heat spreading across the die from junction to case, heat sink, and to ambient.

Examples of such a determination are presented in Fig. 10 because of an experimental value $e^{*}$ of the thermal effusivity $e=\kappa / \alpha^{1 / 2}=\left(\kappa \rho c_{p}\right)^{1 / 2}$ is equal to

$$
e^{*}=\sqrt{C_{\mathrm{th}} / R_{\mathrm{th}}} / S_{\mathrm{a}}^{*} \text {. }
$$

Here, the effective area $S_{\mathrm{a}}{ }^{*}$ depends on design peculiarities of LEDs and is determined by layer sizes and its fill factor $\gamma$, which is practically of 0.4 to.0.7.
Initial lines in Fig. 10 indicate values connected with the thermal parameters of a layer component adjusted initial heat dissipation. Except for Cree XPEHEW LEDs, the whole of the die structures show similar behavior. The Cree XPE-HEW samples have on-substrate from $\mathrm{SiC}$ that serves as an effective heat convector. However, at the thermal time relaxation above $1 \mathrm{~ms}$ heat dissipation occurs to be similar to thermal processes in another LED design types. At these times heat is spreading from junction into unit volume and penetrates up to the interface between DBC and MCPCB layers.

The quantities of the active area $S_{\mathrm{a}}{ }^{*}$ for different LEDs are evaluated with considering the appropriate optical efficiency for the units. Indicated, $e . g$., in Figs. 7(b) and 8(b) effective values $S_{\mathrm{a}}{ }^{*}$ are just directly correlated with geometrical sizes of the layers. In particular, essential bottleneck behavior appeared in Cree XPG dies is manifested at interface DBC and MCPCB near the thermal pad layer. Indeed values of thermal effusivity $e$ and thermal diffusivity $\alpha$ for several layers are listed in Tabl. 1.

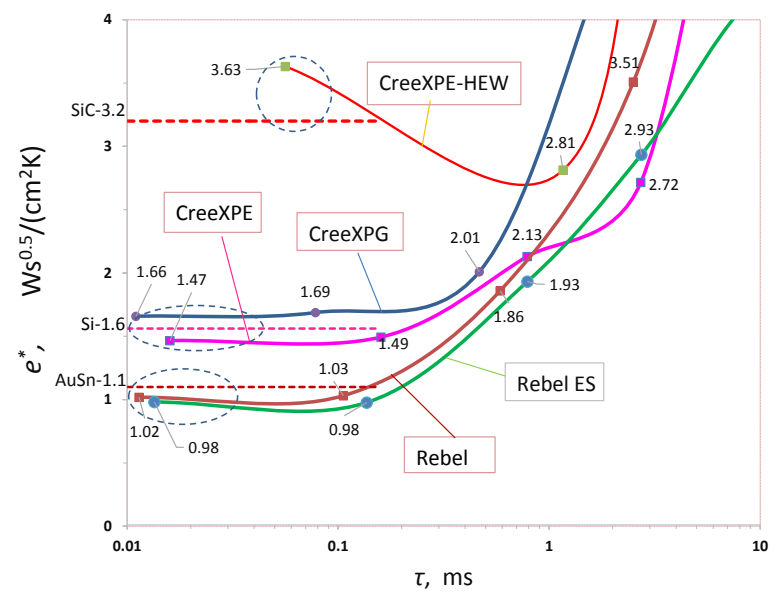

Figure 10: Change in the quantity of $e^{*}$ versus the temporal heat relaxation as characteristic behavior of various types LEDs.

Table 1: Evaluated values of thermal effusivity $e$ and thermal diffusivity $\alpha$ for definite layer components of different LED types.

\begin{tabular}{|l|c|c|}
\hline \multicolumn{1}{|c|}{ Layer material } & $\begin{array}{c}e, \\
\mathrm{Ws}\end{array}$ & $\begin{array}{c}\alpha, \\
\mathrm{cm}^{2} / \mathrm{s}\end{array}$ \\
\hline copper & 3.7 & 1.15 \\
\hline aluminum & 2.4 & 0.91 \\
\hline aluminum alloy & 1.6 & 0.55 \\
\hline silicon carbide $\mathrm{SiC}$ & $2.7-3.5$ & $1.70-2.30$ \\
\hline silicon $\mathrm{Si}$ & $1.5-1.7$ & $0.77-0.90$ \\
\hline AuSn & 1.1 & 0.26 \\
\hline AuSi & 0.8 & 0.13 \\
\hline aluminum nitride $\left.\mathrm{AlN}^{2} \mathrm{~K}\right)$ & $1.8-2.2$ & $0.45-0.68$ \\
\hline aluminum oxide $\mathrm{Al}_{2} \mathrm{O}_{3}$ & $0.8-1.0$ & $0.06-0.09$ \\
\hline
\end{tabular}

Obtained variations in the area $S_{\mathrm{a}}{ }^{*}$ of heat flow across the dies of studied types demonstrate that at the 
interface DBC-MCPCB distinctive thermal kink occurs which is typical for bottleneck behavior $[1,12,13]$ (Fig. 11). Especially, such a feature is the most obvious for Cree XPG and Cree XPE-HEW LEDs. In this case, both types LEDs are constructed on AlN DBC frame.

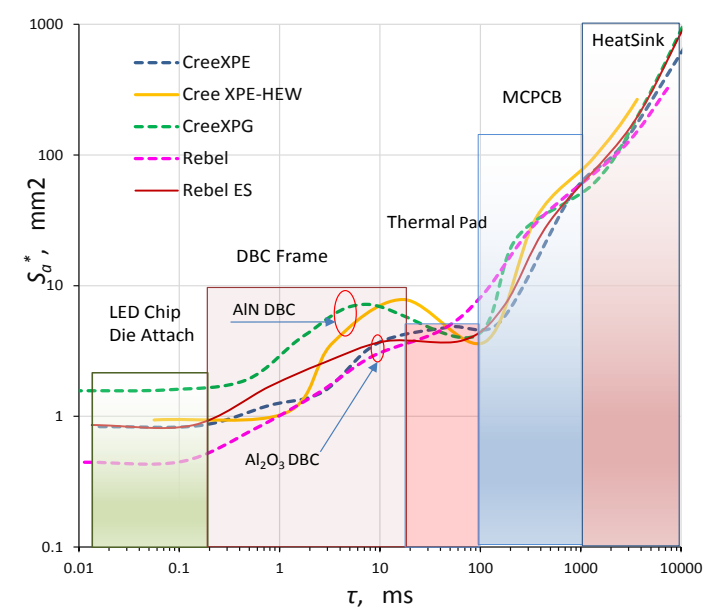

Figure 11: Change in the active area $S_{a}{ }^{*}$ versus the temporal heat relaxation as characteristic behavior of various types of LEDs.

Cumulative structure functions together with $K$ functions demonstrate straightforwardly values of total thermal resistance $R_{\mathrm{ja}}$ from junction to ambient [14] (Fig. 12). Finding the thermal resistance $R_{\mathrm{jc}}$ from junction to case is more indeterminate. Mention that calculated values of $R_{\mathrm{jc}}$ are ordinary received to be underestimated as compared data sheet (Tabl. 2). The method of thermal relaxation spectrometry provides in general more accurate and close quantities of $R_{\mathrm{jc}}$ to data sheet values. The relation $R_{\mathrm{jc}}$ to $R_{\mathrm{ja}}$ occurs to be of 0.2 to 0.5 . More detail data are required on sizes and area of developed LEDs. As a rule, the active area value of the dies is overestimated. It is apparent for Rebel structures, where solder bumps are used, and for sources with VTF structure. Introduced values $S_{\mathrm{a}}{ }^{*}$ are determined by the light efficiency, which depends on the die design and can be rather variable for different LED types.

Table 2: Measured values of thermal resistances of different LED types.

\begin{tabular}{|l|c|c|c|c|}
\hline $\begin{array}{l}\text { LED } \\
\text { types }\end{array}$ & $\begin{array}{c}R_{\mathrm{jc}} \\
\text { (data sheet), } \\
\mathrm{K} / \mathrm{W}\end{array}$ & $\begin{array}{c}R_{\mathrm{jc}} \\
(\mathrm{TTDS}), \\
\mathrm{K} / \mathrm{W}\end{array}$ & $\begin{array}{c}R_{\mathrm{jc}} \\
(\mathrm{T} 3 \mathrm{Ster}), \\
\mathrm{K} / \mathrm{W}\end{array}$ & $\begin{array}{c}R_{\mathrm{ja}}, \\
\mathrm{K} / \mathrm{W}\end{array}$ \\
\hline Rebel & 10 & 9.8 & 10 & 18.9 \\
\hline $\begin{array}{l}\text { Rebel } \\
\text { ES }\end{array}$ & 6 & 6.5 & 6.0 & 18.6 \\
\hline $\begin{array}{l}\text { Cree } \\
\text { XPE }\end{array}$ & 9 & 8.3 & 8.5 & 19.6 \\
\hline $\begin{array}{l}\text { Cree } \\
\text { XPE- } \\
\text { HEW }\end{array}$ & 6 & 5.5 & 4.8 & 17.7 \\
\hline $\begin{array}{l}\text { Cree } \\
\text { XPG }\end{array}$ & 4 & 3.5 & 3.3 & 14.1 \\
\hline
\end{tabular}

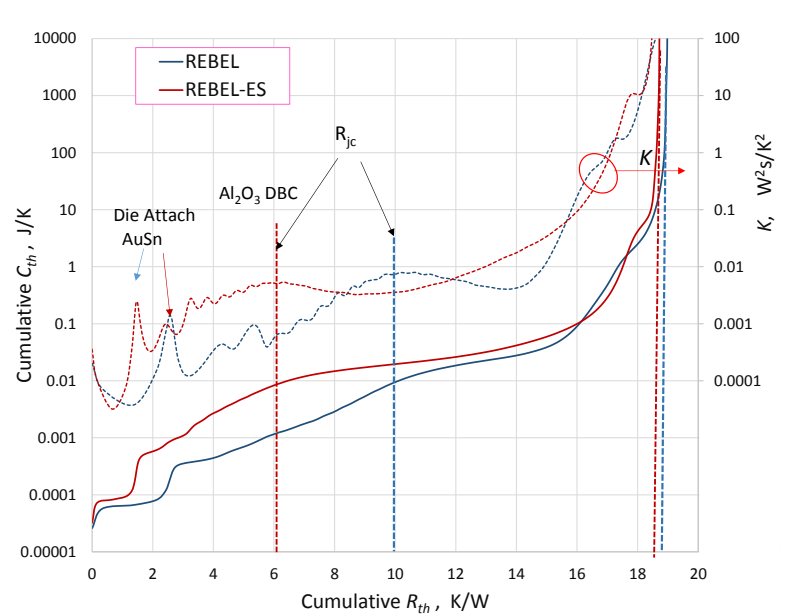

(a)

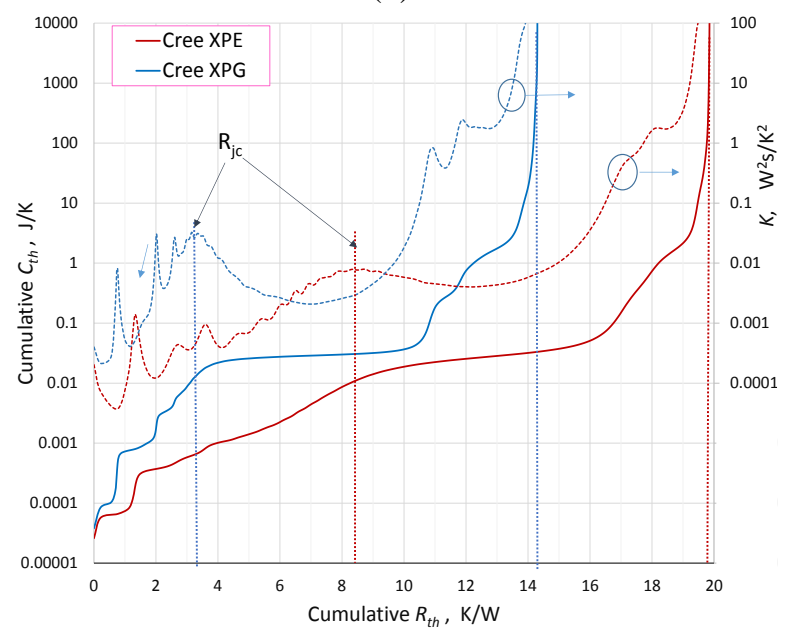

(b)

Figure 12: Determining the thermal resistance $R_{j c}$ and comparison the values (a) for Rebel and Rebel ES and (b) for Cree XPE and Cree XPG units.

Comparative values of the thermal resistances show that the main contribution into the total thermal resistance of different LEDs related with interface between DBC and MCPCB layers (Fig. 13). For Cree XPE-HEW and Cree XPG units this contribution reaches up to $40 \%$ and above. In such design structures benefits obtained due to application of ceramic substrate from AlN DBC are failed since the interface at MCPCB layer results in additional extended thermal resistance.

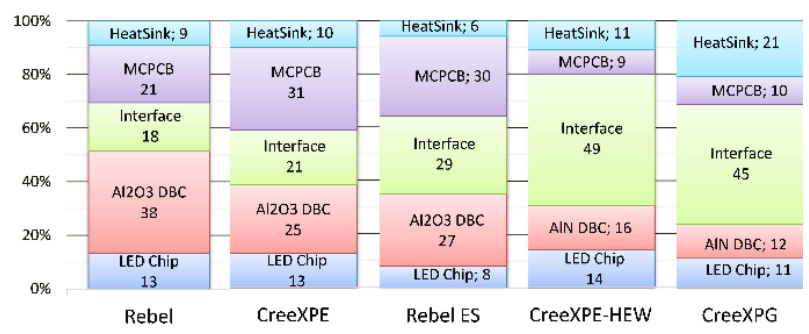

Figure 13: Diagram of relative contribution of the thermal resistances connected with various component layers in the structures of Rebel and Cree LEDs. 


\section{Conclusions}

Analysis of the thermal resistance profile of high efficiency power LEDs of Cree and Luxeon Rebel types is developed. Thermal components of the diodes including thermal resistance, thermal capacitance, and thermal time constant are determined and several distinguishes between different methods are obtained. The value of LED active junction area is evaluated and characteristic behavior of bottleneck effect revealed for definite die configurations and packages.

\section{Acknowledgments}

The work was partly supported by Presidium of the RAS: Basic Research Program No. 24.

\section{References}

1. Pardo, B., Gasse, A., Fargeix, A., Jakovenko, J., Werkhoven, R. J., Perpiña, X., Van Weelden, T., and Bancken, P., "Thermal Resistance Investigations on New Leadframe-based LED Packages and Boards," $13^{\text {th }}$ Int. Conf. on Thermal, Mechanical and Multi-Physics Simulation and Experiments in Microelectronics and Microsystems, EuroSimE 2012, Cascais, 2012, pp. 1/9-9/9.

2. Zakgeim, A. L., Kuryshev, G. L., Mizerov, M. N., Polovinkin, V. G., Rozhansky, I. V., and Chernykov, A. E., "Investigation of Thermal Processes in High-Power AlInGaN Flip-Chip LEDs Using IR-Thermovision Microscopy," Semiconductors, Vol.44, No. 3 (2010), pp. 390396.

3. Bumai, Yu. A., Vaskou, A. S., and Kononenko, V. K., "Measurement and Analysis of Thermal Parameters and Efficiency of Laser Heterostructures and Light-Emitting Diodes," Metrology and Measurement Systems, Vol. 17, No. 1 (2010), pp. 39-46.

4. Pape, H., Schweitzer, D., Chen, L., Kutscherauer, R., and Walder, M., "Development of a Standard for Transient Measurement of Junction-To-Case Thermal Resistance," $12^{\text {th }}$ Int. Conf. on Thermal, Mechanical and Multiphysics Simulation and Experiments in Microelectronics and Microsystems, EuroSimE 2011, Linz, 2011, pp. 1/8-8/8.

5. Bumai, Yu. A., Vaskou, A. S., Kononenko, V. K., and Lomako, V. M., "Relaxation Impedance Spectrometer of Thermal Processes," Electron. info, No. 3 (2010), pp. 58-59.

6. Vaskou, A. S., Kononenko, V. K., and Niss, V. S., "Thermal Relaxation Spectrometry Method and Determining the Parameters of Light-Emitting Diodes," Dokl. BGUIR, No. 4 (2011), pp. 74-79.

7. Stout, R. P. and Billings, D. T., "Accuracy and Time Resolution in Thermal Transient Finite Element Analysis," 2002-Int-ANSYS-Conf-91, http://ansys.com/staticassets/ANSYS/.
8. Carslaw, H. S. and Jaeger, J. C., Conduction of Heat in Solids. Clarendon Press, Sec. Ed. (Oxford, 1959).

9. Bagnoli, P. E., Casarosa, C., Ciampi, M., and Dallago, E., "Thermal Resistance Analysis by Induced Transient (TRAIT) Method for Power Electronic Devices Thermal Characterization - Part I: Fundamentals and Theory," IEEE Trans. Power Electron., Vol. 13, No. 6 (1998), pp. 1208-1219.

10. Glavanovics, M. and Zitta, H., "Thermal Destruction Testing: an Indirect Approach to a Simple Dynamic Thermal Model of Smart Power Switches," Proc. ESSIRC (2001), pp. 236-239.

11. Pape, H., Schweitzer, D., Chen, L., Kutscherauer, R., and Walder, M., "Development of a Standard for Transient Measurement of Junction-to-Case Thermal Resistance," Microelectron. Reliability, Vol. 52, No. 7 (2012), pp. 1272-1278.

12. Liu, Z., Liu, S., Wang, K., and Luo, X., "Status and Prospects for Phosphor-based White LED Packaging," Front. Optoelectron. China, Vol. 2, No. 2 (2009), pp. 119-140.

13. Yan, B., You, J. P., Tran, N. T., He, Y., and Shi, F. G., "Influence of Die Attach Layer on Thermal Performance of High Power Light Emitting Diodes," IEEE Trans. Compon. Packag. Technol., Vol. 33, No. 4 (2010), pp. 722-727.

14. Schweitzer, D., Pape, H., and Chen, L., "Transient Measurement of the Junction-to-Case Thermal Resistance Using Structure Functions: Chances and Limits," Proc. $24^{\text {th }}$ SEMI-THERM Sympos., San Jose, 2008, pp. 193-199. 